\title{
LAS DECISIONES DE INVERSIÓN DE LOS INDIVIDUOS
}

\author{
Beatriz Herrera García \\ Docente de la Facultad de Ciencias Contables
}

\begin{abstract}
RESUMEN
Los modelos sobre los determinantes de los precios de los activos -como variables clave en los mercados por su capacidad de revelar información-y, en definitiva, sobre la valoración del riesgo, surgen de los enfoques alternativos, de los cuales el de técnica de equilibrio, descansa en el análisis tradicional de demanda y oferta, el comportamiento optimizador de los agentes económicos y vaciado de mercado. Dicha técnica tiene ventajas importantes en mercados y contextos donde las fricciones institucionales son relevantes, sin embargo, es necesario adoptar supuestos especializados sobre la naturaleza de las preferencias de los individuos o sobre los procesos estocásticos de las variables económicas claves.

En éste tipo de modelos de valoración de activos, los agentes económicos, dotados cada uno de unas cantidades iniciales de cada bien o activo financiero, intercambian entre sí de acuerdo con sus preferencias y de forma de lo que se desea vender de cada bien en agregado sea igual a lo que se desea comprar. Las proporciones en que se intercambian los bienes son unas constantes que coinciden con la tasa a que cada agente está dispuesto a intercambiar cada par de bienes. Estas constantes reciben el nombre de precios de equilibrio.
\end{abstract}

Palabras clave: Activos financieros, selección de carteras, preferencias.

\section{INTRODUCCIÓN}

Comprender el comportamiento inversionista de los individuos o, si se quiere, de los agentes económicos, es suponer (según el enfoque tradicional de la Teoría Económica) la existencia y los gustos de dichos agentes económicos como exógenos a la teoría. Sin embargo, éste enfoque tradicional no se hace extensivo a las empresas ${ }^{1} \mathrm{o}$ intermediarios financieros ${ }^{2}$. La existencia de estas organizaciones económicas se entiende fundamentalmente en base a las funciones que sirven, en lugar de funcionar principalmente porque existen.

En cualquier caso, para desarrollar las funciones de estas organizaciones es necesario estudiar y derivar, en primer lugar, el comportamiento de los agentes económicos o individuos. Por lo que resulta conveniente dividir la decisión de inversión por parte de los individuos en los siguientes componentes:

1 En el análisis detallado dela valoración de activos financieros haciendo uso explícito delas preferencias y funciones de utilidad de los individuos, las empresas, poseen, activos físicos de producción para la economíay emiten activos financieros para financiarsus actividades de producción.

2 Los intermediarios financieros son poseedores yemisores de activos financieros, que invierten solamenteen forma indirecta en activos físicos o reales. 
1. Decisión de consumo-ahorro, en la cual los individuos deciden la cantidad óptima de su riqueza actual que debe asignarse a consumo presente y la cantidad que debe destinarse a inversión para poder así consumir en el futuro.

2. Selección de carteras, ${ }^{3}$ en la que el individuo decide cómo colocar sus ahorros entre las diversas alternativas que ofrece el conjunto de oportunidades de inversión.

En este contexto nos enfrentamos a un problema de asignación óptima de nuestros recursos en un ambiente intertemporal y entre varias alternativas, y para que dicho problema esté bien definido, debe suponerse que el individuo tiene un orden de preferencias mediante el cual establece un ranking de las posibles alternativas de elección ${ }^{4}$.

\section{TEORIA DE LA UTILIDAD ESPERADA}

En contextos de incertidumbre es posible llegar a la representación de las preferencias de los agentes económicos mediante la llamada utilidad esperada (es una forma de representar las preferencias de los agentes más restrictiva que el uso de las funciones ordinales de utilidad). La ventaja es que este nuevo criterio permite interpretar mejor la toma de decisiones de los agentes.

Siempre es posible representar las preferencias de un individuo sobre un activo financiero a través de una relación de preferencias o a través de la utilida a esperada. Este resultado fue inicialmentz demostrado por Von Neumann Morgenstern, por lo que se le denomina "Utilidad esperada de Von NeumannMorgensterny. En el modelo se supone que el agente decidor tiene una determinada función de utilidad sobre su consumo, $\mathrm{U}(\mathrm{C})$. que conoce la probabilidad de consegur determinados niveles de consumo y que elegirá entre distintas alternativas max1mizando la utilidad esperada de su consumo.

La utilidad esperada viene dada por la ecuación:

$$
E U(C)=\sum_{i=1}^{n} \pi . U(C)
$$

donde:

$\pi$. denota la probabilidad que el agente asigna a un determinado nivel de consumo.

C. denota un determinado nivel de consumo.

EU(C) es la utilidad esperada.

Es de observar que la incertidumbre asociada al consumo es una variable distinta a la utilidad del consumo. E.g., en caso de dos posibles niveles de consumo, $C$. con probabilidad $\pi$ y $C$, con probabilidad $(1-\pi)$, el consumo esperado, $\mathrm{E}(\mathrm{C})$, la varianza del consumo, $\operatorname{Var}(\mathrm{C})$, y la utilidad esperada del consumo vienen dados por:

$$
\begin{gathered}
E(C)=\pi C_{1}+(1-\pi) C_{2} \\
\operatorname{Var}(C)=\pi\left[C_{1}-E(C)\right]^{2}+(1-\pi)\left[C_{2}-E(C)\right]^{2} \\
\left.E U(C)=\pi U\left(C_{1}\right)+(1-\pi) U C_{2}\right)
\end{gathered}
$$

Donde, por un lado, se puede hablar del riesgo (o su variabilidad) del consumo en sí (algo que se puede medir a través de la

3 Combinación de activos que produce pagos futuros no negativos. A los inversores sólo les preocupa el rendimiento esperado (su media) y la varianza de dichos rendimientos cuando se ven obligados a discriminar entre inversiones alternativas. La varianza la podemos describir comola variabilidad que experimentan los rendimientos (precios) de un activo alrededor de su media.

4 Orden de preferencias que satisface los axiomas de la utilidad esperada de Von Neumann-Morgenstern. 
varianza del consumo), y por otro, de la actitud del agente frente al riesgo (algo que viene definido por su función de utilidad). Mientras que el riesgo es un concepto que depende de las características específicas del activo financiero que se considera, la actitud frente al riesgo va depender del individuo se sí, y por lo tanto, puede ser distinta para distintos agentes.

De hecho dependiendo de la forma de la función de utilidad, se puede distinguir hasta tres tipos de actitudes frente al riesgo.

La primera, cuando la primera derivada de la función de utilidad es positiva $\left[\mathrm{U}^{\prime}(\mathrm{C})\right.$ $>0]$, por lo que la función de utilidad es creciente en el consumo. En este caso, la segunda derivada es negativa por lo que una unidad extra de consumogenera más utilidad para niveles bajos de consumo que para niveles altos de consumo; la utilidad marginal del consumo es decreciente. Siempre se supone que los agentes tienen funciones de utilidad de este tipo, es decir, que son aversos al riego.

La segunda, cuando la primera derivada de la función de utilidad es positiva [ $\mathrm{U}^{\prime}(\mathrm{C})$ $>0]$, y la segunda derivada es igual a cero $\left[\mathrm{U}^{\prime \prime}(\mathrm{C})=0\right]$, la función de utilidad es creciente y lineal. En este caso, la utilidad marginal es positiva y constante. Los agentes que tienen funciones de utilidad de este tipo, se dice que tiene neutralidad frente al riesgo (neutral al riesgo). En el caso de neutralidad, la disminución en la utilidad cuando el consumo cae es igual al aumento en utilidad cuando el consumo se incrementa. Así, la utilidad esperada es la misma en ambos casos.

La tercera, cuando la primera derivada de la función de utilidad es positiva $\left[\mathrm{U}^{\prime}(\mathrm{C})>0\right.$, y la segunda detivada de dicha función es también positiva [U"(C)>0]. En este caso, la utilidad marginal es positiva y creciente, es decir, unidades sucesivas de consumo dan lugar a mayores aumentos de utilidad. Los agentes que tienen funciones de utilidad de esta tipo, se dice que tienen propensión al riesgo (propenso o amante al riesgo). En el caso de propensión al riesgo, la caída en utilidad al disminuir el consumo es más pequeña que el aumento en utilidad cuando el consumo se incrementa, de forma que el individuo está mejor con riesgo que sin riesgo.

Analicemos con un poco más detalle el caso más habitual de aversión al riesgo (primer tipo de actitud frente al riesgo), y veamos como se asocia la aversión al riesgo a las funciones de utilidad.

\begin{tabular}{|c|c|c|}
\hline $\begin{array}{c}\text { Unidades consumidas de } \\
\text { un mismo bien }\end{array}$ & Utilidad total & Utilidad marginal \\
1 & 2.0 & 2.0 \\
2 & 3.4 & 1.4 \\
3 & 4.0 & 0.6 \\
4 & 4.4 & 0.4 \\
& & \\
\hline
\end{tabular}


varianza del consumo), y por otro, de la actitud del agente frente al riesgo (algo que viene definido por su función de utilidad). Mientras que el riesgo es un concepto que depende de las características específicas del activo financiero que se considera, la actitud frente al riesgo va depender del individuo se sí, y por lo tanto, puede ser distinta para distintos agentes.

De hecho dependiendo de la forma de la función de utilidad, se puede distinguir hasta tres tipos de actitudes frente al riesgo.

La primera, cuando la primera derivada de la función de utilidad es positiva [ $\mathrm{U}^{\prime}(\mathrm{C})$ $>0]$, por lo que la función de utilidad es creciente en el consumo. En este caso, la segunda derivada es negativa por lo que una unidad extra de consumogenera más utilidad para niveles bajos de consumo que para niveles altos de consumo; la utilidad marginal del consumo es decreciente. Siempre se supone que los agentes tienen funciones de utilidad de este tipo, es decir, que son aversos al riego.

La segunda, cuando la primera derivada de la función de utilidad es positiva $\left[\mathrm{U}^{\prime}(\mathrm{C})\right.$ $>0$ ], y la segunda derivada es igual a cero $\left[\mathrm{U}^{\prime \prime}(\mathrm{C})=0\right]$, la función de utilidad es creciente y lineal. En este caso, la utilidad marginal es positiva y constante. Los agentes que tienen funciones de utilidad de este tipo, se dice que tiene neutralidad frente al riesgo (neutral al riesgo). En el caso de neutralidad, la disminución en la utilidad cuando el consumo cae es igual al aumento en utilidad cuando el consumo se incrementa. Así, la utilidad esperada es la misma en ambos casos.

La tercera, cuando la primera derivada de la función de utilidad es positiva $\left[\mathrm{U}^{\prime}(\mathrm{C})>0\right.$ ], y la segunda derivada de dicha función es también positiva $\left[\mathrm{U}^{\prime \prime}(\mathrm{C})>0\right.$ ]. En este caso, la utilidad marginal es positiva y creciente, es decir, unidades sucesivas de consumo dan lugar a mayores aumentos de utilidad. Los agentes que tienen funciones de utilidad de esta tipo, se dice que tienen propensión al riesgo (propenso o amante al riesgo). En el caso de propensión al riesgo, la caída en utilidad al disminuir el consumo es más pequeña que el aumento en utilidad cuando el consumo se incrementa, de forma que el individuo está mejor con riesgo que sin riesgo.

Analicemos con un poco más detalle el caso más habitual de aversión al riesgo (primer tipo de actitud frente al riesgo), y veamos como se asocia la aversión al riesgo a las funciones de utilidad.

\begin{tabular}{|c|c|c|}
\hline $\begin{array}{c}\text { Unidades consumidas de } \\
\text { un mismo bien }\end{array}$ & Utilidad total & Utilidad marginal \\
1 & 2.0 & 2.0 \\
2 & 3.4 & 1.4 \\
3 & 4.0 & 0.6 \\
4 & 4.4 & 0.4 \\
\hline
\end{tabular}


Veamos ahora cómo elige este individuo entre distintos niveles de consumo.

Consumo 1. Este nivel de consumo ofrece al individuo un consumo $\mathrm{C}=2$ con certidumbre (es decir, con probabilidad 1). El consumo medio, la varianza del consumo y la utilidad esperada ofrecidos son:

$$
\mathrm{E}(\mathrm{C})=2 ; \operatorname{Var}(\mathrm{C})=0 ; \quad \mathrm{EU}(\mathrm{C})=3.4
$$

Consumo 2. Este nivel de consumo ofrece un nivel de consumo 1 con una probabilidad del $50 \%$ y un nivel de consumo 3 con una probabilidad del $50 \%$.

$$
\begin{gathered}
\mathrm{E}(\mathrm{C})=0,5 \times 1+0,5 \times 3=2 \\
\operatorname{Var}(C)=0,5(1-2)^{2}+0,5(3-2)^{2}=1 \\
\mathrm{EU}(\mathrm{C})=0,5 \times \mathrm{U}(1)+0,5 \times \mathrm{U}(3)=0,5 \times 2+0,5 \times 4=3
\end{gathered}
$$

Comparando estos niveles de consumo se aprecia que, aunque ambas ofrecen el mismo consumo medio, de acuerdo con un criterio de utilidad esperada, el individuo estrictamente prefiere la primera a la segunda. La razón es que la segunda ofrece el mismo consumo en promedio, pero con una mayor varianza. Por eso se llama «averso al riesgo» a un individuo con estas prefe-rencias. Comparando ambos casos, la mitad de las veces el consumo ha disminuido de 2 a una unidad, entonces la utilidad decrece en 1,4. La otra mitad de las veces, el consumo aumenta de 2 a 3 unidades, pero esto provoca solamente un aumento de 0.6 en utilidad. Así, aunque en promedio el consumo es el mismo, la caída en utilidad provocada por caídas en el consumo no se ve compensada por el aumento en utilidad provocadas por subidas en el consumo.

\section{LOS PROBLEMAS BÁSICOS Y TOMA DE DECISIONES}

Según la Teoría Económica los prea que surgen de los mercados competitproporcionan a sus participantes infemación útil para realizar un proceso de tom de decisiones. En particular, los mercaa: de capitales hacen que los precios res tantes reflejen en forma conjunta $\mathbf{b}$ información disponible y las expectativas as la gran variedad de participantes en $1 t$ mercados.

Sobre la evidencia de la teoría de las mercados eficientes, se puede afirmar que esa información conjunta contien. predicciones precisas sobre los sucesic! futuros que las que cualquier grupo o agente económico individual pueda obtener por 5 propia cuenta. De esta forma los mercad: : de capitales realizan una labor importanie al poner a disposición pública una información superior no alcanzable porotrc : medios ${ }^{5}$.

Asimismo, sugieren que en mercador: financieros desarrollados, la tasa de inflacion esperada de corto plazo puede deducirse ae los tipos corrientes de los Bonos del teson: En la misma línea, es conocido que los precios de la Bolsa de Valores tienden a ser unos excelentes indicadores de la actividad económica general. $\mathrm{Y}$ según evidencias empíricas, las diferencias en los tipos de interés que ofrecen las obligaciones emitidas por distintas empresas contienen información sobre las posibilidades de quiebra de las mismas.

Por ejemplolos precios a futuro en mercados de bienes contienen un estimador del precio «spot» que dicho bien tendrá en el futuro. Esa información es importantes a la hora de decidir la cantidad a producir y almacenar del mencionadoproducto. 
La estructura temporal de los tipos de interés - la diferencia de los tipos de interés que se obtienen para periodos de inversión a largo plazo y los que se reciben en inversiones con una menor duración temporal-puede utilizarse para estimar los tipos que prevalecerán en el futuro y permite una política monetaria más fundamentada. En las Bolsas, las opciones de compra y venta reflejan en sus precios un estimador implícito de la incertidumbre asociada con los precios de los activos financieros sobre los que se están negociando dichas opciones.

De lo anterior, se asume que las finanzas debe aprender a revelar consistentemente predicciones económicas mediante la observación de los precios de los activos financieros que surgen de los mercados competitivos. Esta es la base de la eficiencia informática de los mercados financieros y de los fundamentales problemas que la información asimétrica, entre los individuos, plantea al estudio de las finanzas. Por ello los economistas financieros construyen modelos teóricos sobre los factores determinantes de los precios de los activos financieros basándose en el comportamiento macroeconómico racional-maximización de la utilidad esperada sujeta a una determinada restricción- o, alternativamente en planteamientos más generales que se fundamentan en la ausencia de posibilidades de arbitraje ${ }^{6}$.

En resumen, en este campo de actuación de las finanzas como es la determinación de los precios de los activos financieros han sido creados importantes bancos de datos financieros con los que los modelos teóricos han podido ser expuestos a sofisticados contrastes empíricos.

\section{REGULACIÓN DE LOS INTER- MEDIARIOS FINANCEROS Y DE LOS MERCADOS DE CONTRA- TACIÓN DE AC'TIVOS FINAN- CIEROS}

Debido a la posibilidad de emitir y comprar activos directa o indirectamente a través de los intermediarios financieros en los mercados de contratación correspondientes, los individuos pueden alterar y adecuar su consumo de bienes y servicios de acuerdo con sus preferencias a lo largo del tiempo, así como redistribuir los riesgos que conllevan las fluctuaciones del valor de los activos físicos o reales. En definitiva, los mercados y los intermediarios financieros que en ellos actúan permitan transferir riqueza a lo largo del tiempo de forma que sus preferencias de consumo se vean satisfechas, ofreciendo además la necesaria liquidez para que resulte posible dirigir sus recursos hacia las inversiones más rentables con rapidez y facilidad.

También debido a la posibilidad de emitir activos financieros, las empresas son capaces de obtener capital por diversas fuentes. Así, los mercados permiten obtener una gran cantidad de recursos de una gran diversidad de individuos de forma que se facilite la posibilidad de emprender proyectos de gran envergadura. Los mercados de capitales, cargando implícitamente a las empresas diferentes precios, se convierten en un medio de asignar los recursos escasos entre diversos

- Cualquier estrategia de inversión que permita ganar dinero a cambio de nada es una estrategia de arbitraje. Por otro lado, se dice que para que no exista la posibilidad de realizar una oportunidad de arbitraje debe ser cierto que si un activo financiero ouna combinación de activos-cartera-producepagos futurosnonegativos, entonces, necesariamente el coste de dicho activo o cartera en el momento de realizar la inversión debe ser también no negativo. 
usos competitivos. Así, los intermediarios financieros competitivos y los mercados de capitales desarrollados ofrecen una importante información a través de los precios.

Es decir, mediante préstamos o gracias a la posibilidad de prestar, un individuo puede elegir entre retrasar o acelerar el consumo personal de su riqueza disponible. Invirtiendo en activos financieros relativamente seguros, un individuo puede transmitir una mayor carga de riesgos hacia otros individuos. Sin embargo, dado que los inversores son, muy probablemente y en promedio, aversos al riesgo, solamente pueden verse inducidos a aceptar mayores riesgos bajo la promesa de un rendimiento esperado mayor. De esta forma, los mercados de capitales ofrecen una gran flexibilidad en ajustar los riesgos de las inversiones con las preferencias y opiniones de una enorme variedad de individuos. Asimismo, no solamente los riesgos se redistribuyen entre los individuos a través de los mercados gracias a la esperada compensación en términos de mayores rendimientos, sino que incluso es posible eliminar, hasta cierto punto, riesgos mediante una diversificación adecuada, o mediante estrategias de cobertura apropiadas.

Asimismo, los precios de los activos financieros que reflejan información sobre la futura situación de las empresas juegan también un importante papel en la asignación de recursos entre ellas. Precios altos motivan a las empresas a aumentar su capital propio. Incluso más importante es que dichos precios se transformen en señales que reflejan la aprobación o rechazo por parte de los mercado de las acciones que las empresas toman. Así, las preferencias de los inversores y la agregada sabiduría del mercado, se ponen a disposición de los directivos empresariales.

\section{ALGUNAS CONCLUSIONES SOBRE LA EFICIENTE TOMA DE DECISIONES}

Ante las interrogantes tradicionales. debido principalmente a la incertidumbre asociada al problema de los tipos de proyectos de inversión que deben ser aceptados y, a las ventajas de los recursos ajenos - deuda-sobre los recursos propios -acciones-a la hora de financiar inversiones. La discusión tradicional se centra en el impacto que la legislación fiscal tiene sobre las decisiones de financiación de las empresas. En este sentido, cabe señalar la ventaja que puede suponer para la financiación mediante deuda, la posibilidad de deducir los intereses asociados a dichas emisiones de recursos ajenos. Naturalmente, se refrere a las ventajas relativas sobre la financiación mediante recursos propios ya que, al contrario que los intereses, los dividendos no son deducibles. Estas ventajas potenciales deben contraponerse con los posibles peligros de insolvencia que un excesivo endeudamiento podria tener para la empresa.

Por el lado de los conflictos de intereses que surgen entre los agentes económicos participantes en las empresas: accionistas, acreedores y gerentes, y el diseño óptimo de contratos que minimicen dichos conflictos, se ha convertido en un área crucial de estudio dentro del problema caracterizado por la toma eficiente de decisiones empresariales. Los posibles costos que surgen como consecuencia de los conflictos entre los intereses alternativos de los participantes reciben el nombre de «costes de agencia». Se pueden presentar conflictos 
entre los accionistas y los gerentes, siempre que estos últimos no poseen el cien por ciento del capital. El gerente puede actuar, en estos casos, dirigiendo parte de su esfuerzo a transferir recursos hacia su propia persona al no participar de la totalidad de beneficios pero soportando, por otra parte, íntegramente los costos asociados con su actividad. Esta transferencia de recursos que pueden utilizar los gerentes en su propio interés puede aminorarse emitiendo deuda. Así, la empresa compromete unos pagos futuros dejando un menor margen de maniobra a los gerentes. No olvidar, que los acreedores tienen prioridad sobre los accionistas en el cobro de los recursos generados en la empresa. Este es un beneficio asociado con la financiación mediante recursos ajenos.

Por otro lado, los conflictos que surgen entre los accionistas y los acreedores incentivan a los primeros a llevar a cabo inversiones no óptimas. Para entender esta idea, es preciso reconocer que los recursos propios son equivalentes a una opción de compra sobre los activos de la empresa. En general, una opción de compra otorga el derecho, no la obligación, a comprar un número determinado de acciones a un precio establecido denominado precio de ejercicio. Si elvalor de esas acciones en una fecha futura, previamente determinada, es superior al precio de ejercicio, el titular de la opción ejercerá su derecho ${ }^{7}$. Así, el valor de esa opción en el momento de su vencimiento será:

$$
\mathrm{C}_{\mathrm{T}}=\max \left[0, \mathrm{P}_{\mathrm{T}}-\mathrm{K}\right]
$$

Donde:

$\mathrm{C}_{\mathrm{T}}$ es el valor de la opción de compra

$\mathrm{P}_{\mathrm{T}}$ es el valor de la acción en el momento

del vencimiento de dicha opción

$\mathrm{K}$ es el precio de ejercicio

En la ecuación anterior se puede observar que cuanto mayor sea la variabilidad del precio futuro de la acción, mayor valor tendrá la opción ya que su propietario tiene una mayor probabilidad de que el precio de la acción termine por encima del precio de ejercicio.

Ahora, supongamos una empresa que tiene una determinada cantidad de deuda con un valor nominal igual a $\mathrm{D}$ y unos recursos propios cuyos propietarios reciben el valor residual una vez compensados los inversores en recursos ajenos. $\mathrm{Al}$ vencimiento de la deuda, si X, la cantidad de recursos generados por los activos reales de la empresa, es suficientemente elevada, los accionistas recibirán $\mathrm{X}-\mathrm{D}$, en caso contrario no percibirán absolutamente nada pero, dado que existe la responsabilidad limitada, tampoco se verán obligados a poner dinero de su propio bolsillo para compensar en su totalidad a los acreedores. En suma dichos accionistas tienen un pago final al vencimiento de la deuda igual a:

$$
\begin{aligned}
& \mathrm{P}_{\mathrm{T}}=\max [0, \mathrm{X}-\mathrm{D}] \\
& \text { Donde: }
\end{aligned}
$$

$\mathrm{P}_{\mathrm{T}}$ es el valor de la acción en el momento de vencimiento de dicha opción.

Si esta ecuación se utiliza en términos de opciones, es evidente que dicha

En caso contrario, también aumentala posibilidad de que el precio de la acción acabe por debajodel preciode ejercicio, pero en esta caso, el propietario dela opción sencillamente no ejerce su derecho, no viéndoseobligado a experimentar una pérdida más allá del precio pagado por la opción en su momento. 
estructura de pagos finales corresponde a una opción de compra sobre los activos de la empresa, siendo $\mathrm{D}$ el precio de ejercicio.

En efecto, desde el momento que los recursos propios son como una opción de compra en los activos de la empresa, los accionistas tienen incentivos, como propietarios, a invertir en proyectos de alto riesgo; esto es, en proyectos con una alta variabilidad en sus pagos futuros. Naturalmente este comportamiento es anticipado con facilidad por los acreedores que transfieren sus costos a los propios accionistas a través de las características contractuales de la deuda. Por tanto, este es el coste asociado con la financiación mediante recursos ajenos. La estructura óptima de capital es una consecuencia del beneficio y del coste de dichas emisiones de deuda empresarial.

En definitiva, las decisiones de inversión que realizan los individuos se pueden clasificar en inversiones reales e inversiones financieras. Por inversiones reales se entiende la creación de un activo físico de larga duración. Así, la construcción de una nueva planta, un nuevo producto o la puesta en marcha de una nueva red de distribución de los productos de la empresa, entre muchos otros, servirán como ejemplos. Por el contrario, las inversiones financieras se producen cuando se adquiere un derecho sobre una inversión real ya existente. Así, la propiedad de las acciones de una empresa otorgan el derecho a recibir unos beneficios futuros en forma de ganancias (pérdidas) de capital y dividendos. En consecuencia, las empresas tiene que financiar sus inversiones reales, y la forma habitual de hacerlo es emitir unos títulos (acciones, bonos, warrants, obligaciones convertibles, etc.), que los inversores individuales $\mathrm{e}$ institucionales comprar. Claro está, la propiedad de dichos títulos otorga a los individuos que los compran unos derechos sobre los recursos (inciertos) que generarán en el futuro dichas inversiones reales. Naturalmente, el mayor o menor grado de riesgo que incorporan dichas inversiones hará que la compensación exigida por los individuos para aceptar dicha inversión sea diferen65te en cada caso. Lo importante es que al tomar la decisión en un momento dado el inversor debe conocer, o más exactamente, tener una estimación racional dada toda la información disponible de lo que valen en ese preciso monumento los derechos adquiridos.

\section{REFERENCIAS}

ALIAGA VÁLDEZ, C. y ALIAGA CALDERÓN, C., Matemáticas financieras. Un enfoque práctico, Prentice Hall, Colombia, 2002.

BLACK, Leland y TARQUÍN, Anthony, Ingenieria Económica, Mc Graw Hill, Colombia, 1999.

BREALEY, R. and S. Myers, Principles of Corporate Finance, Mc Graw Hill, 2000.

HERRERA, B., "Las decisiones de financiamiento de capital", Revista Quipukamayoc, Vol. 12, N. ${ }^{\circ}$ 24, 2005.

MARÍN, J. and R. Rahi, "Information Revelation and Market Incompleteness", Review of Economic Studies 67, 2000.

ROSS, Westerfield y Jaffe, Finanzas corporativas, Mc Graw Hill, México, 2000.

ZVI Bodie y MERTON, Robert C. (Premio Nobel de Economía 1997), Finanzas, Prentice Hall, México, 1999. 